\title{
Altered White Matter Microstructure in the Corpus Callosum and Its Cerebral Interhemispheric Tracts in Adolescent Idiopathic Scoliosis: Diffusion Tensor Imaging Analysis
}

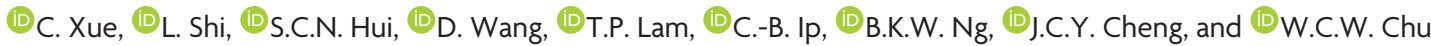

\begin{abstract}
BACKGROUND AND PURPOSE: Neural system was one of the important contributors to the etiopathogenesis of adolescent idiopathic scoliosis; additionally, the morphology of corpus callosum interconnecting both hemispheres of the brain was found to be altered morphologically. Our aim was to evaluate and compare the microstructural changes of the corpus callosum and its interhemispheric white matter fiber tracts interconnecting both cerebral hemispheres in patients with adolescent idiopathic scoliosis and matched controls using diffusion tensor imaging.
\end{abstract}

MATERIALS AND METHODS: Brain DTI was performed in 69 patients with adolescent idiopathic scoliosis (female, right thoracic/thoracolumbar curve) and 40 age-matched controls without adolescent idiopathic scoliosis (female). 2D and 3D segmentation of the corpus callosum were performed using a region-growing method, and the corpus callosum was further divided into 6 regions, including the rostrum, genu, anterior and posterior midbodies, isthmus, and splenium. The laterality index was calculated to quantify the asymmetry of the corpus callosum. Interhemispheric fiber tractography were performed using the Brodmann atlas.

RESULTS: 2D ROI analysis revealed reduced fractional anisotropy in the genu and splenium $(P=.075$ and $P=.024$, respectively). Consistently reduced fractional anisotropy on the left sides of the genu and splenium was also found in 3D ROI analysis ( $P=.03$ and $P=.012$, respectively). The laterality index analysis revealed a pseudo-right lateralization of the corpus callosum in adolescent idiopathic scoliosis. Interhemispheric fibers via the splenium interconnecting Brodmann 3, 1, and 2; Brodmann 17; and Brodmann 18 (corresponding to the primary somatosensory cortex and primary and secondary visual cortices) were also found to have reduced fractional anisotropy $(P \leq .05)$.

CONCLUSIONS: Reduced fractional anisotropy was found in the genu and splenium of the corpus callosum and corresponding interhemispheric fiber tracts interconnecting the somatosensory and visual cortices via the splenium. Our results are suggestive of altered white matter microstructure within the brain of those with adolescent idiopathic scoliosis, which could be related to abnormal brain maturation during adolescence in adolescent idiopathic scoliosis and could possibly explain the previously documented somatosensory function impairment and visuo-oculomotor dysfunction in this condition.

ABBREVIATIONS: AIS = adolescent idiopathic scoliosis; $C \mathrm{CC}=$ corpus callosum; $\mathrm{FA}=$ fractional anisotropy; $\mathrm{LI}=$ laterality index

A dolescent idiopathic scoliosis (AIS) is the most common form of scoliosis that causes 3D structural deformities of the spine, which affect approximately $4 \%$ of teenagers during pu-

Received September 22, 2017; accepted after revision February 14, 2018.

From the Departments of Imaging and Interventional Radiology (C.X., L.S., S.C.N.H. D.W., C.-B.I., W.C.W.C.) and Orthopedics and Traumatology (T.P.L., B.K.W.N., J.C.Y.C.), Prince of Wales Hospital, The Chinese University of Hong Kong, Shatin, New Territories, Hong Kong.

This work was supported, in part, by grants from the Research Grants Council of the Hong Kong Special Administrative Region (Project No. 14206716 and SEG_CUHK02) and the Fondation Yves Cotrel de l'Institut de France.

Please address correspondence to Dr Lin Shi for technical issues and W.C.W. Chu for clinical issues, Department of Imaging and Interventional Radiology, Prince of Wales Hospital, The Chinese University of Hong Kong, Sha Tin, N.T., Hong Kong SAR; e-mail: shilin@cuhk.edu.hk (L. Shi); winniechu@cuhk.edu.hk (W.C.W. Chu)

- Indicates open access to non-subscribers at www.ajnr.org

http://dx.doi.org/10.3174/ajnr.A5634 berty. AIS has been reported to involve impaired balance control, ${ }^{1}$ possibly due to a combination of disruptive somatosensory ${ }^{2}$ and visuo-oculomotor and vestibular ${ }^{3}$ functions. Multiple studies have repeatedly shown evidence of disturbed somatosensory function in AIS, with a higher percentage of abnormal cortical somatosensory-evoked potentials compared with healthy controls. ${ }^{1,2}$

Apart from the neurophysiologic dysfunction, the neuromorphologic changes in AIS have been documented by various imaging studies with changes in the cord ${ }^{4,5}$ and brain, including low cerebellar tonsils, ${ }^{6}$ abnormalities in white matter density, ${ }^{7}$ cerebral cortical thickness, ${ }^{8}$ and corpus callosum (CC) morphology. ${ }^{9,10}$ Asymmetries in the cerebral hemispheres ${ }^{7,9,11}$ and vestibular apparatus ${ }^{12}$ were also found in AIS. The neural system is proposed as one of the important contributors to the etiopathogenesis of AIS. ${ }^{13}$

AJNR Am J Neuroradiol 39:1177-84 Jun 2018 www.ajnr.org 
Diffusion tensor imaging visualizes water diffusion of white matter fiber tracts. ${ }^{14}$ The fractional anisotropy (FA) value is commonly applied to interpret the altered microstructure of these tracts. ${ }^{14}$ It is highly sensitive to microstructural changes and could be used to reflect microstructural integrity such as fiber density, axonal diameter, and myelination of white matter.

A previous DTI study of the spinal cord and hindbrain showed lower FA values in the medulla oblongata and cervical spinal cord. ${ }^{4}$ Another DTI study by Joly et $\mathrm{al}^{15}$ found a significant decrease in FA values in the midbody of the CC, which possibly suggests abnormal brain development in AIS.

The objective of this study was to use the DTI technique in a larger subject cohort to investigate whether there is any difference in white matter microstructure within the brain between AIS and matched controls using a 3D approach. The ROI was focused on the CC because it is the largest fiber bundle and connector between the 2 cerebral hemispheres.

\section{MATERIALS AND METHODS \\ Subjects}

The research protocol was approved by the Clinical Research Ethics Committee of the institution and was conducted in compliance with the principles of the Declaration of Helsinki. Written informed consent was obtained from all subjects. Subjects with AIS and healthy controls (age- and sex-matched) were prospectively recruited for DTI from January 2012 to December 2014. Patients with AIS were recruited from our outpatient clinic, while the healthy controls were recruited from local schools. All participants were right-handed Chinese female adolescents 12-18 years of age. Inclusion criteria of patients with AIS were typical scoliosis curves convex to the right side. They were carefully assessed clinically to rule out other known associated causes of scoliosis and any neurologic abnormalities. Healthy controls were screened by experienced orthopedic surgeons to exclude minor scoliosis. Exclusion criteria of all subjects were a history of head or back injury, developmental delay, or psychiatric illness.

\section{MR Imaging Acquisition}

All subjects underwent MR imaging using a 3T scanner (Achieva TX series; Philip Healthcare, Best, the Netherlands) equipped with an 8-channel sensitivity encoding head coil. For the suppression of eddy current artifacts, brain sensitivity encoding-DTI was acquired using a single-shot EPI sequence with the following parameters: $\mathrm{TR}=8667 \mathrm{~ms}, \mathrm{TE}=60 \mathrm{~ms}, \mathrm{FOV}=224 \times 224 \mathrm{~mm}^{2}$, flip angle $=90^{\circ}$, NEX $=1$, matrix $=112 \times 109$, sections $=70$, section thickness $=2 \mathrm{~mm}$, no interslice gap with an in-plane image resolution $=2 \times 2 \mathrm{~mm}$. Thirty-two directions of diffusion gradients with b-values of $1000 \mathrm{~s} / \mathrm{mm}^{2}$ and $1 \mathrm{~B}_{0}$ volume were collected. Conventional T1-weighted images of the brain for mapping were obtained using a 3D fast-field echo imaging sequence with following parameters: $\mathrm{TR}=18 \mathrm{~ms}, \mathrm{TE}=2.4 \mathrm{~ms}$, FOV $=210 \times 210 \mathrm{~mm}^{2}$, flip angle $=30^{\circ}$, NEX $=1$, matrix $=$ $232 \times 232$, sections $=200$.

\section{MR Image Preprocessing}

The FMRIB Software Library (FSL; http://www.fmrib.ox.ac.uk/ fsl) ${ }^{16}$ was used to perform eddy current correction and head mo- tions correction by registering each data point to the first $b=0$ image with affine transformation. Nonbrain tissues were also excluded using the FSL Brain Extraction Tool (http://fsl.fmrib.ox. ac.uk/fsl/fslwiki/BET). ${ }^{16}$ After the preprocessing methods, all data were manually checked to exclude any errors.

\section{Voxelwise Statistical Analysis}

For voxelwise statistical analysis, the Tract-Based Spatial Statistics (TBSS; http://fsl.fmrib.ox.ac.uk/fsl/fslwiki/TBSS) pipeline available in FSL 5.0.9 was performed. ${ }^{17}$ First, FA maps from all participants were smoothed using Gaussian filters with the full width at half maximum of $6 \mathrm{~mm}$ and aligned into a $1-\mathrm{mm}^{3}$ FA template created by aligning every FA image to every other one using the FMRIB Nonlinear Registration Tool (FNIRT; http://fsl.fmrib.ox. ac.uk/fsl/fslwiki/FNIRT). Second, the mean FA image was calculated from all normalized FA images and thinned to create a mean FA skeleton, which represented the centers of all tracts common to the group. Finally, the skeleton was set to have an FA threshold of 0.2 ( $F A \geq 0.2$ ) to ensure that gray matter regions were excluded from the analyses. Resulting skeletonized data were fit into voxelwise cross-subject statistics. The FSL Randomise method (https:// fsl.fmrib.ox.ac.uk/fsl/fslwiki/Randomise) was used with threshold-free cluster enhancement at a $P$ value $<.05$ to compare the data and correct the multiple comparisons.

\section{ROI Analysis}

After we obtained the FA maps, a 2D segmentation of the CC was performed manually in the midsagittal plane as shown in Fig $1 A$, followed by a $3 \mathrm{D}$ segmentation of the same region semiautomatically using a region-growing method in the FA maps by ITKSNAP 2.4 (www.itksnap.org). ${ }^{18}$ For consistency, all 3D segmented CCs consisted of 15 slices on either side of the midlines and the midline image (ie, 31 slices in total), which incorporated the main bulk of CCs as illustrated in Fig $1 B$. All segmented CCs were manually checked for correctness. Regional segmentation of CCs was performed on the basis of the template defined by Witelson, ${ }^{19}$ which included the rostrum, genu, anterior midbody, posterior midbody, isthmus, and splenium from an anterior-toposterior direction. 3D segmentation was further divided into left (LHS) and right (RHS) sides, with the midsagittal line acting as the dividing line. The laterality index $(\mathrm{LI})^{20}$ was calculated by the following formula:

$$
L I=\frac{\text { Mean } F A_{\mathrm{LHS}}-\text { Mean } F A_{\mathrm{RHS}}}{\text { Mean } F A_{\mathrm{LHS}}+\text { Mean } F A_{\mathrm{RHS}}} .
$$

The laterality index shows left hemispheric lateralization by the value $\geq+0.1$, while the value $\leq-0.1$ indicates rightward asymmetry. Values between +0.1 and -0.1 represent bilateral lateralization. $^{20}$

\section{DTI Tractography Analysis}

DSI Studio (http://dsi-studio.labsolver.org/) ${ }^{21}$ was used to process the corrected diffusion-weighted images for fiber tractography analysis. To measure and quantify the fiber tracking from the left to right hemisphere of the brain, we applied the Brodmann atlas, ${ }^{22}$ and the midsagittal line was used as the middle line to separate the atlas into left and right sides. The Brodmann atlas was 

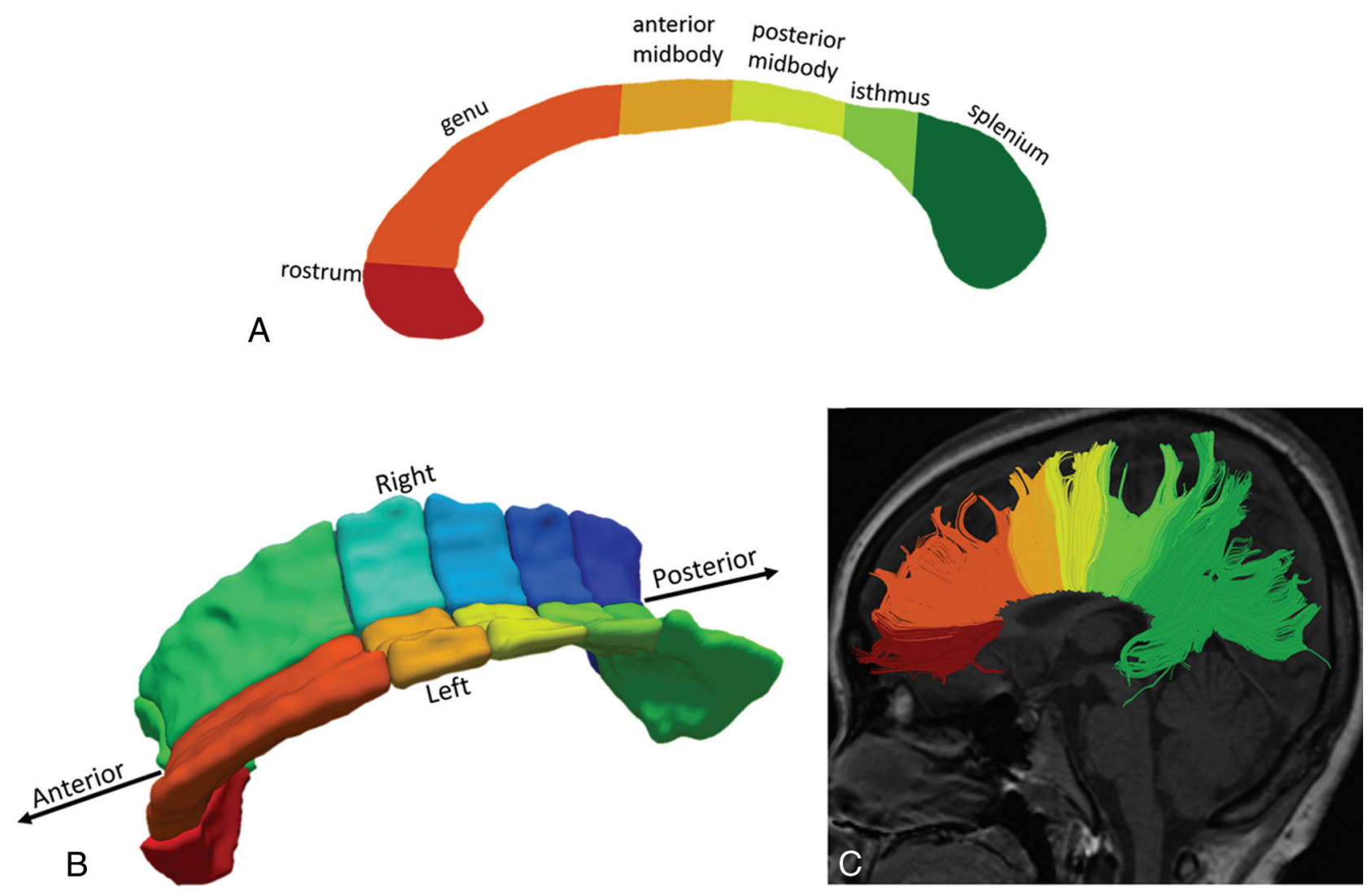

FIG 1. Subdivision of the CC based on the Witelson template ${ }^{19}$ in the midsagittal view in 2D segmentation $(A)$, 3D segmentation (B), and fiber tractography $(C)$.

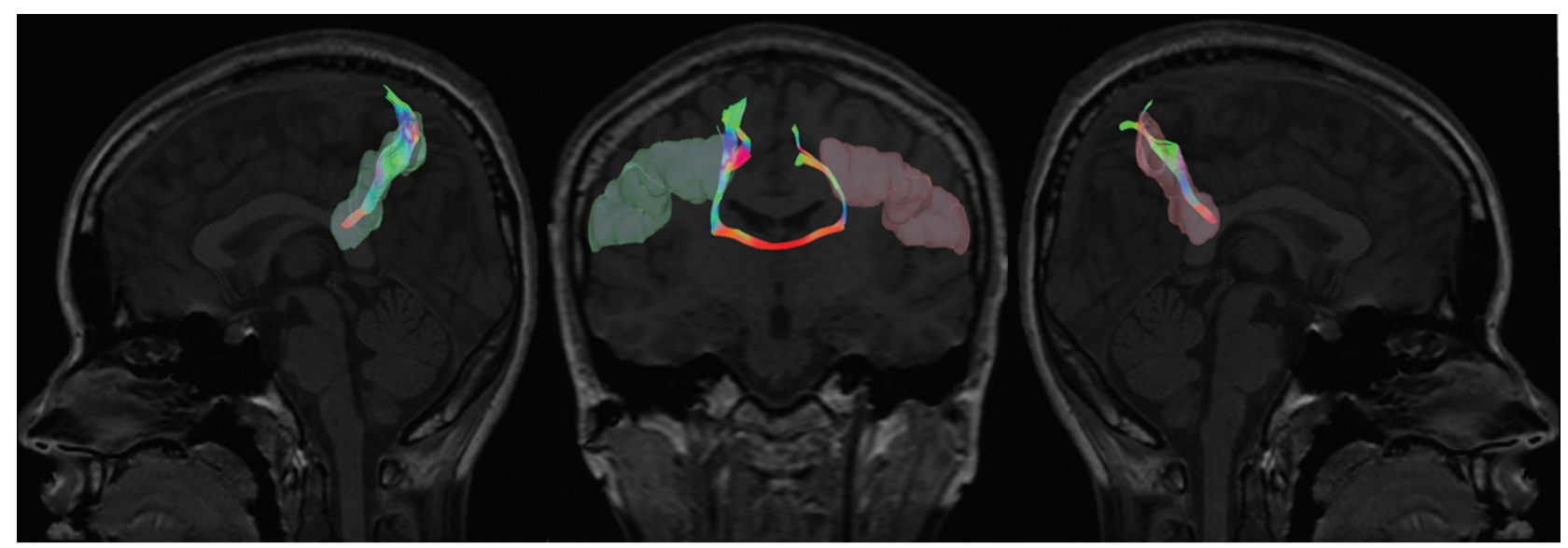

FIG 2. Illustration of the interhemispheric tract with the ROls (left and right Brodmann 2) selected from the Brodmann atlas.

then registered nonlinearly to the subject space using the statistical parametric mapping (SPM software; http://www.fil.ion. ucl.ac.uk/spm/software/spm12) nonlinear registration algorithm. For each tract, left and right ROIs were placed to establish the tract network with the following cutoffs and thresholds: FA threshold $=0.15$; angular threshold $=70,{ }^{23}$ only limited to fibers passing through the CC as shown in Fig 2.

\section{Statistics}

The FA maps in the AIS group were compared with those in the control groups using an independent $t$ test. One-way ANOVA was performed to compare mean FA values among the control and
AIS groups with different levels of severity (ie, mild, moderate, and severe) with Bonferroni as a post hoc analysis. The level of statistical significance was defined at $P \leq .05$. False discovery rate correction was used for multiple comparison corrections.

\section{RESULTS}

\section{Participant Characteristics}

Sixty-nine right-handed patients with AIS (female; mean age, $14.5 \pm 2.2$ years; mean Cobb angle, $\left.33.55^{\circ} \pm 13.9^{\circ}\right)$ and 40 agematched right-handed healthy controls (female; mean age, $14.6 \pm$ 1.04 years) were recruited. The AIS group consisted of 65 patients with a dominant right thoracic curve and 4 patients with a dom- 
Table 1: Demographics of subjects with AIS with different curve severities and healthy controls

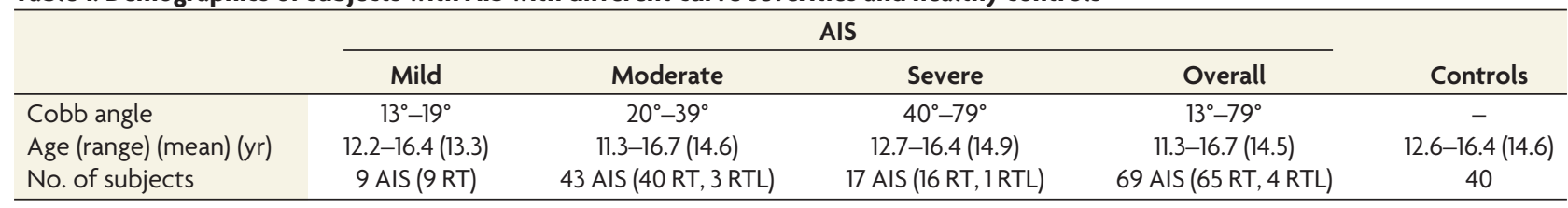

Note:-RT indicates right thoracic; RTL, right thoracolumbar.

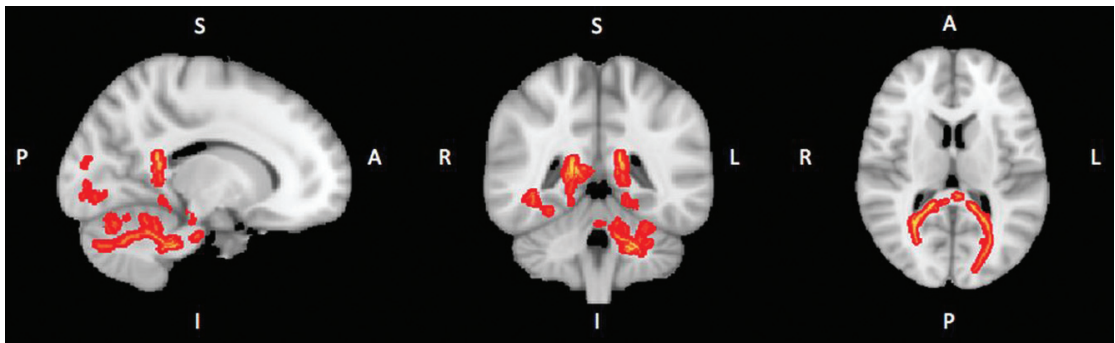

FIG 3. Results of voxelwise statistical analysis using the TBSS pipeline. The red lines show the lower FA value in the voxels in the subject with AIS compared with control subjects. $S$ indicates superior; I, inferior; R, right; L, left; P, posterior; A, anterior.

inant right thoracolumbar curve. Their demography is shown in Table 1 .

The 69 patients with AIS were further divided into 3 groups based on the severity of their scoliosis curve defined by the Cobb angle as follows: mild group $\left(n=9\right.$, Cobb angle $\left.=13^{\circ}-19^{\circ}\right)$; moderate group $\left(n=43\right.$, Cobb angle $\left.=20^{\circ}-39^{\circ}\right)$; and severe group $\left(n=17\right.$, Cobb angle $\left.=40^{\circ}-79^{\circ}\right) .^{5} \mathrm{~T} 1$ - and T2-weighted images were reviewed by an experienced radiologist (W.C.W.C.), and no structural brain abnormality was found in any subject.

\section{Voxel-Based Analysis}

Using the TBSS analysis implemented in FSL, we found no significant lower FA $(P=.438)$ between the 2 groups voxelwise, even though slightly lower mean FAs were found in the splenium of patients (Fig 3).

\section{D ROI Analysis of the Corpus Callosum}

The summary of FA values at different regions of the CC from 2D analysis is shown in Fig 4. Significantly lower mean FA values in patients with AIS were found in the genu (uncorrected $P=.025$ ) and splenium (uncorrected $P=.004$ ). Compared with different scoliosis severities, significantly lower FA values were found only in the splenium (corrected $P=.02$ ), while a marginal difference (corrected $P=.069$ ) of lower FA values was observed in the genu when we compared the control with the moderate group. After multiple comparison correction, significantly reduced FA values were persistently found in the splenium of patients with AIS (corrected $P=.024)$. The overall summary is shown in Table 2 .

\section{D ROI Analysis of the Corpus Callosum}

Using 3D segmentation, we performed analysis of the CC in detail with data obtained from the whole region instead of only 1 midsagittal slice in 2D. We also conducted the left-right comparison, which was not performed in the previous $2 \mathrm{D}$ study. ${ }^{15}$

FA values were compared between the left and right sides of the CC, summarized in Fig 5 and Table 3. Lower FA values were found significantly on the left side of splenium (corrected $P=$
.012 ) and in the left genu (corrected $P=$ $.03)$. Comparisons among different severities of AIS revealed significantly lower FA values on the left side of the splenium (corrected $P=.048$ ) and genu (corrected $P=.012$ ) in the moderate group. FA values from the left side of the splenium (corrected $P=.078$ ) also showed a trend toward lower values in the severe group.

The LI assessed lateralization of the brain. Comparison between controls and patients with AIS is shown in Fig 6 and Table 4. Results indicated that LI values in different regions of the CC of both those with AIS and controls fell between +0.1 and -0.1 , compatible with bilateral lateralization in both subject groups, however, with a significantly lower LI in the splenium of the corpus callosum (corrected $P=.012$ ) and in the genu (corrected $P=.03$ ) of those with AIS. The above findings reflected significant interhemispheric FA asymmetry of the CC with reduced FA on the left side (pseudo-right lateralization) in AIS compared with controls. This pseudo-right lateralization was also significant (corrected $P<.05$ ) when comparing controls with the moderate AIS group.

\section{DTI Tractography Analysis}

The mean FA values of all the resulting tracts were extracted and compared between patients with AIS and controls. Significantly lower FA values were only observed in Brodmann 3, 1, and 2, with mean FA values of 0.517 (AIS) versus 0.53 (controls) $(P=.045)$, which reflect the primary somatosensory cortex; Brodmann 17 with mean FA values of 0.567 (AIS) versus 0.581 (controls) ( $P=$ .027 ); and Brodman 18 with mean FA values 0.571 (AIS) versus 0.582 (controls) $(P=.038)$, which are correlated with the primary visual cortex (V1) and secondary visual cortex (V2), correspondingly, in AIS. When we compared patients with AIS with different severities, lower FA values were also detected in Brodmann 17 with a mean FA value of $0.563(P=.007)$ and Brodmann 18 with a mean FA value of $0.5693(P=.029)$ in moderate cases.

\section{DISCUSSION}

The corpus callosum is known as the largest white matter fiber bundle connecting both hemispheres of the brain. ${ }^{24}$ Different segments of the CC are connected to different cerebral regions ${ }^{24}$ as shown in Fig 1C. Topographic organization of the CC is supported by various studies ${ }^{25,26}$ in patients with surgical resection of callosal lesions: Anterior callosal axons transfer motor information between frontal lobes, while posterior fibers crossing the posterior midbody, isthmus, and splenium transfer somatic sensory, auditory, and visual information. ${ }^{26}$ 


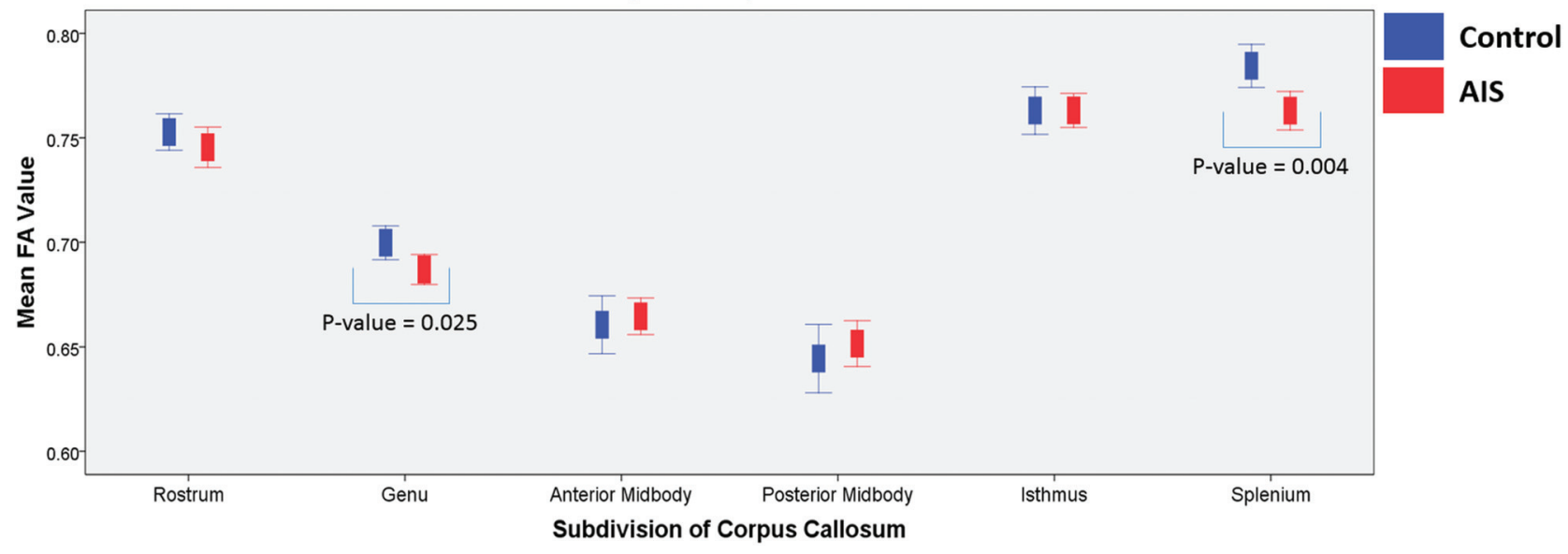

FIG 4. Summary of FA values of each subdivision of the corpus callosum divided by the Witelson template $\mathrm{e}^{19}$ in the $2 \mathrm{D}$ segmentation ROI analysis. Blue represents controls, and red represents patients with AIS.

Table 2: Descriptive statistics of the FA comparison between controls and patients with AIS in $2 \mathrm{D}$ segmentation ${ }^{\mathrm{a}}$

\begin{tabular}{lllcc}
\hline & \multicolumn{1}{c}{ Controls } & \multicolumn{1}{c}{ AIS } & $\begin{array}{c}\boldsymbol{P} \text { Value } \\
\text { (Uncorrected) }\end{array}$ & $\begin{array}{c}\boldsymbol{P} \text { Value } \\
\text { (Corrected) }\end{array}$ \\
\hline Rostrum & $0.7528 \pm 0.02735$ & $0.7455 \pm 0.04039$ & .31 & .62 \\
Genu & $0.6997 \pm 0.02552$ & $0.6870 \pm 0.02955$ & .025 & .08 \\
Anterior midbody & $0.6607 \pm 0.04330$ & $0.6647 \pm 0.0366$ & .61 & .73 \\
Posterior midbody & $0.6445 \pm 0.05131$ & $0.6516 \pm 0.04586$ & .46 & .68 \\
Isthmus & $0.7630 \pm 0.03550$ & $0.7631 \pm 0.03340$ & .99 & .99 \\
Splenium & $0.7845 \pm 0.03228$ & $0.7630 \pm 0.03855$ & .004 & .02 \\
\hline
\end{tabular}

${ }^{a}$ Data are means unless otherwise indicated.

${ }^{\mathrm{b}} \mathrm{P}$ value after multiple comparison correction.

In our 2D ROI analysis, significantly lower FA values were found in the genu and splenium. In 3D ROI analysis, significantly lower FA values at the left genu and left splenium were consistently found as in the 2D ROI analysis, while in the study of Joly et $\mathrm{al},{ }^{15}$ lower FA values were found in the midbody. The above apparent discrepancies could be partially explained by different templates used in dividing the CC. The genu in this study overlapped the anterior midbody in the study of Joly et al. Hence, both studies were basically in agreement; however, an additional area of lower FA value was found in the splenium of patients with AIS in the current study, which might be partially explained by differences in subject recruitment. Our study included a larger number of patients with AIS with major right thoracic curves while in the study of Joly et al, subjects predominantly had major thoracolumbar curves.

The genu of the CC provides interconnection to the prefrontal cortex, premotor cortex, and supplementary motor area. ${ }^{24}$ Caille et $\mathrm{al}^{25}$ studied patients without epilepsy with resection of different portions of the anterior CC, showing that motor coordination transfer occurred at the level of the middle portion of the genu. Our study showed a significantly lower FA value and reduced laterality index in the genu of patients with AIS, which concurs with previous fMRI findings about an abnormal activation in the supplementary motor area and a significantly higher interhemispheric asymmetry index in the premotor cortex and supplementary motor area in patients with AIS. ${ }^{27}$

Our tractography analysis found lower FA values in the fibers passing through the splenium and interconnecting primary somatosensory cortex (Brodmann 3, 1 , and 2) and the primary and secondary visual cortices (Brodmann 17 and 18) in patients with AIS. Fabri et $\mathrm{al}^{28}$ performed an fMRI study in a human subject who had undergone resection of the CC (callosotomy) in 2 stages and concluded that integrity of the posterior body of the CC/splenium was required for normal interhemispheric transfer of somatosensory input to activate the related cerebral cortex. In the current study, we found significantly lower FA values on the left side of the splenium after multiple corrections. Our findings correspond with the clinical observation of somatosensory-evoked-potential dysfunction in AIS. ${ }^{1,2,29}$ According to the study of Fabri et al, ${ }^{28}$ for subjects with an intact posterior body of the CC, peripheral somatosensory stimulation leads to activation of both contralateral and ipsilateral somatosensory areas and the posterior parietal cortex; however, if the posterior half and splenium of the CC was damaged, activation could only be observed in the contralateral cortex. In this study, lower FA was found on the left side, which corresponded to the reported somatosensory-evoked-potential abnormalities $^{29}$ found over the right cerebral cortex in patients with AIS with a typical right thoracic scoliotic curve.

Other reported studies have provided evidence that transfer of visual $^{30,31}$ information between the hemispheres occurs in the splenium. Our finding of reduced FA values in fibers interconnecting the visual cortex coincides with findings of a previous study in which smaller brain volume closely related to the visual cortex was found in patients with AIS. ${ }^{9}$ Moreover, visually impaired children have been shown to have a higher risk of spinal deformity. $^{32}$

What is the possible link between such alterations of the CC and scoliosis development in AIS? The human brain develops extensively during adolescence, with maturation of the CC continuing until early adulthood, ${ }^{33}$ characterized by both myelination and axonal pruning; hence, development of the brain could be reflected by FA values in DTI because it reveals information 


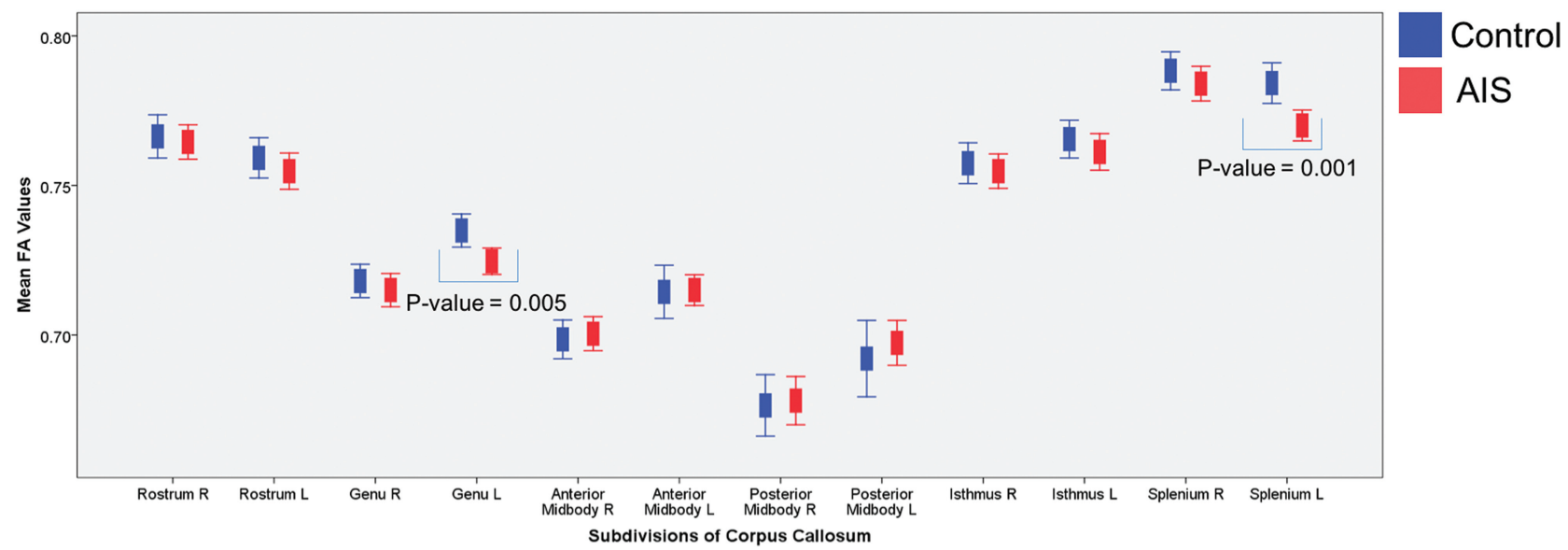

FIG 5. Summary of the mean FA values on the right and left of 3D segmentation of the CC for each subdivision according to the Witelson template $^{19}$ in controls and patients with AIS.

Table 3: Descriptive statistics of the FA comparison between controls and patients with AIS in 3D segmentation ${ }^{\mathrm{a}}$

\begin{tabular}{|c|c|c|c|c|c|c|c|c|}
\hline & \multicolumn{2}{|c|}{ Controls } & \multicolumn{2}{|c|}{ AIS } & \multicolumn{2}{|c|}{$\begin{array}{c}P \text { Value } \\
\text { (Uncorrected) }\end{array}$} & \multicolumn{2}{|c|}{$\begin{array}{c}P \text { Value } \\
\text { (Corrected) }\end{array}$} \\
\hline & LH & RH & LH & RH & LH & RH & LH & RH \\
\hline Rostrum & $0.7592 \pm 0.02106$ & $0.7664 \pm 0.02260$ & $0.7548 \pm 0.02522$ & $0.7645 \pm 0.02396$ & .33 & .69 & 1 & .83 \\
\hline Genu & $0.7349 \pm 0.01735$ & $0.7180 \pm 0.0175$ & $0.7246 \pm 0.01843$ & $0.7073 \pm 0.02316$ & $.005^{c}$ & .47 & $.03^{\mathrm{c}}$ & .94 \\
\hline Anterior midbody & $0.7144 \pm 0.02782$ & $0.6985 \pm 0.02027$ & $0.7150 \pm 0.02142$ & $0.7004 \pm 0.02379$ & .91 & .67 & .91 & .90 \\
\hline Posterior midbody & $0.6921 \pm 0.03998$ & $0.6764 \pm 0.03216$ & $0.6973 \pm 0.03141$ & $0.6780 \pm 0.03358$ & .48 & .81 & .82 & .88 \\
\hline Isthmus & $0.7655 \pm 0.01978$ & $0.7574 \pm 0.02134$ & $0.7612 \pm 0.02547$ & $0.7548 \pm 0.02395$ & .36 & .56 & .87 & .85 \\
\hline Splenium & $0.7842 \pm 0.02128$ & $0.7883 \pm 0.01990$ & $0.7701 \pm 0.02154$ & $0.7841 \pm 0.02404$ & $.001^{c}$ & .34 & $.012^{c}$ & 1 \\
\hline
\end{tabular}

Note:- LH indicates left-hand side; RH, right-hand side.

${ }^{a}$ Data are means unless otherwise indicated.

b $P$ value after multiple comparison correction.

c Statistically significant $(P<.05)$.

Left Lateralization

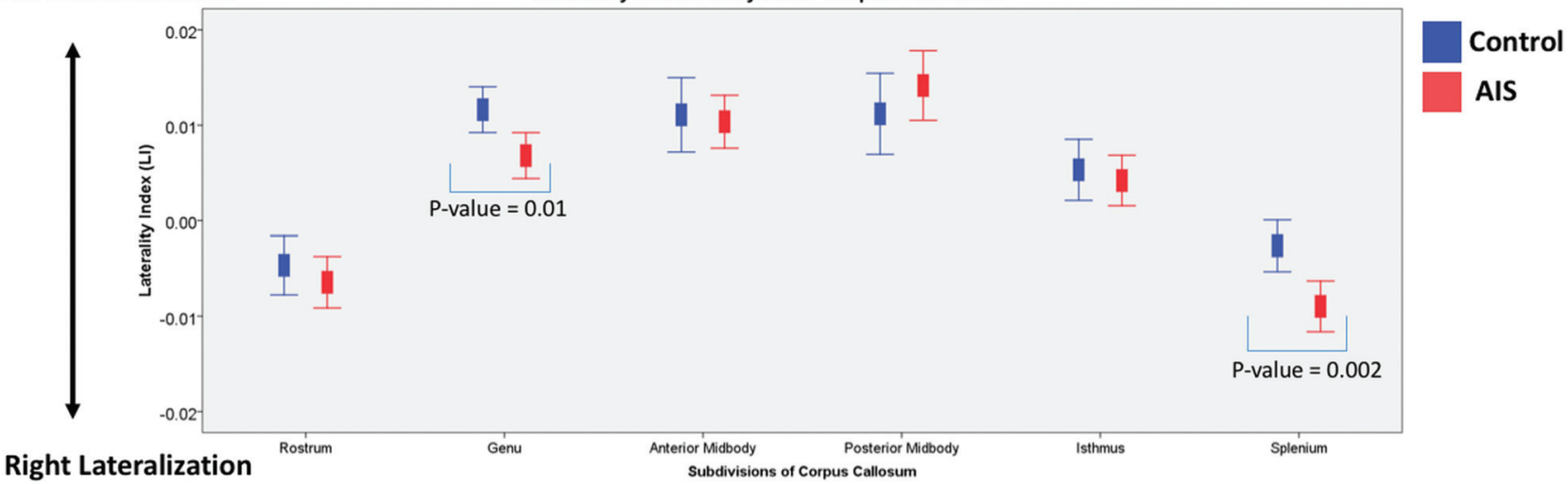

FIG 6. The laterality index of the CC for both patients with AIS (red) and controls (blue). The laterality index was calculated by the following formula: $L I=\left(\right.$ Mean $F A_{\mathrm{LHS}}-$ Mean FA $\left._{\mathrm{RHS}}\right) /\left(\right.$ Mean $F A_{\mathrm{LHS}}+$ Mean $\left.F A_{\mathrm{RHS}}\right)$.

about the homogeneity of water diffusion. Different regions of the CC mature at different pubertal stages. ${ }^{34}$ Both the genu and splenium become more mature and thicker, reaching $90 \%$ of maximal thickness in earlier puberty, ${ }^{34}$ which coincides with the typical period for the onset of the scoliotic curve in AIS. Burwell et $\mathrm{al}^{35}$ have formulated a collective model of the pathogenesis of AIS, which is a neuro-osseous timing of the maturation system operating in a child's internal world during growth and maturation. Burwell et al have suggested that AIS progresses as the neural system fails to maintain a balance of postural equilibrium to con- trol asymmetric growth of a rapidly enlarging and moving adolescent spine. In line with the above theory, we hypothesize that the brain maturation process, including the maturation of the genu and splenium in AIS, might be interrupted during the rapid growing period of adolescence when onset and/or progression of the scoliotic curve occurs. Our finding has suggested that there are alterations of white matter in the CC reflected by lower FA values. These alterations are also in agreement with previously reported morphologic changes of the CC by MR imaging. ${ }^{9,10}$

Furthermore, we found reduced FA on the left side of the CC 
Table 4: Descriptive statistics of the laterality index comparison between controls and patients with AIS

\begin{tabular}{lcccc}
\hline & Controls & AIS & $\begin{array}{c}P \text { Value } \\
\text { (Uncorrected) }\end{array}$ & $\begin{array}{c}P \text { Value } \\
\text { (Corrected) }\end{array}$ \\
\hline Rostrum & $-0.0047 \pm 0.00965$ & $-0.0064 \pm 0.01120$ & .41 & .61 \\
Genu & $0.0116 \pm 0.00750$ & $0.0068 \pm 0.01001$ & $.01^{\mathrm{c}}$ & $.03^{\mathrm{c}}$ \\
Anterior midbody & $0.0111 \pm 0.01220$ & $0.0104 \pm 0.01156$ & .76 & .76 \\
Posterior midbody & $0.0112 \pm 0.01331$ & $0.0142 \pm 0.01521$ & .31 & .61 \\
Isthmus & $0.00053 \pm 0.01001$ & $0.0042 \pm 0.01101$ & .60 & .72 \\
Splenium & $-0.00026 \pm 0.00848$ & $-0.009 \pm 0.01108$ & $.002^{\mathrm{c}}$ & $.012^{\mathrm{c}}$ \\
\hline
\end{tabular}

${ }^{a}$ Data are means unless otherwise indicated.

${ }^{\mathrm{b}} P$ value after multiple comparison correction.

' Statistically significant $(P<.05)$.

involving the genu and splenium in patients with AIS with a dominant right convex scoliotic curve. These observations might not be just a casual phenomenon but could have implications related to underlying spinal asymmetry. The pseudo-right lateralization of the CC might be part of the known generalized asymmetry of many functions and structures proposed in AIS, such as cerebral hemisphere asymmetries, ${ }^{7,9,11}$ vestibular apparatus asymmetries, ${ }^{12}$ extraspinal left-right skeletal length asymmetries, and proximodistal lower limb disproportions (allometry), ${ }^{35}$ though the exact mechanism and clinical significance (whether it is related to primary etiopathogenesis or is a secondary consequence of AIS) is not certain.

One of the limitations of this study is the unequal number of subjects in the mild, moderate, and severe groups of AIS, with most subjects belonging to moderate group. This is the genuine distribution of AIS in the daily clinical setting and probably explains why a significant difference is consistently observed in the moderate group only, while the other subgroups, though showing similar trends, have not reached the significance level. For future studies, a concerted effort of multiple centers with recruitment of more patients with mild and severe AIS would be useful to further investigate the relationship between those alterations in the CC and the effect on scoliosis curve severity.

The neuropsychologic dysfunctions of AIS are usually subclinical, though they can be elicited by specific tests; however, these tests are sophisticated and expensive and have not been included in this study, which is another limitation. Examples of these specific tests, however, have been well-documented in the published literature, which include video-oculography for visuooculomotor dysfunction $^{3}$ and electromyographic analysis for gait $^{36}$ in AIS.

Although there is a significant FA difference in the CC and related cerebral regions, reflecting white matter microstructural changes, the FA values derived from DTI do not involve nonGaussian properties of biologic tissues and intravoxel fiber crossings, ${ }^{37}$ which may interfere with FA value accuracy. Diffusional kurtosis imaging, which involves more sophisticated calculations, can overcome the limitations by eliminating some confounding factors. Future studies using the more advanced diffusional kurtosis imaging might be useful to further validate our DTI findings.

\section{CONCLUSIONS}

Reduced FA was found in the genu and splenium in patients with AIS compared with matched controls on both 2D and 3D seg- mentation analysis. There was corresponding reduction of $\mathrm{FA}$ in fibers interconnecting the primary somatosensory cortex and visual cortex, passing through the splenium in patients with AIS. Furthermore, relatively reduced FA was also observed on the left side of the above regions of the CC in patients with AIS presenting with a typical right scoliotic curve. The above microstructural changes of the white matter fiber tracts might be related to the documented somatosensory function impairment and visuo-oculomotor dysfunction in AIS.

\section{REFERENCES}

1. Lao ML, Chow DH, Guo X, et al. Impaired dynamic balance control in adolescents with idiopathic scoliosis and abnormal somatosensory evoked potentials. J Pediatr Orthop 2008;28:846-49 CrossRef Medline

2. Guo X, Chau WW, Hui-Chan CW, et al. Balance control in adolescents with idiopathic scoliosis and disturbed somatosensory function. Spine 2006;31:E437-40 CrossRef Medline

3. Lion A, Haumont T, Gauchard GC, et al. Visuo-oculomotor deficiency at early-stage idiopathic scoliosis in adolescent girls. Spine 2013;38:238-44 CrossRef Medline

4. Kong Y, Shi L, Hui SC, et al. Variation in anisotropy and diffusivity along the medulla oblongata and the whole spinal cord in adolescent idiopathic scoliosis: a pilot study using diffusion tensor imaging. AJNR Am J Neuroradiol 2014;35:1621-27 CrossRef Medline

5. Chu WC, Man GC, Lam WW, et al. Morphological and functional electrophysiological evidence of relative spinal cord tethering in adolescent idiopathic scoliosis. Spine 2008;33:673-80 CrossRef Medline

6. Chu WC, Man GC, Lam WW, et al. A detailed morphologic and functional magnetic resonance imaging study of the craniocervical junction in adolescent idiopathic scoliosis. Spine 2007;32:1667-74 CrossRef Medline

7. Shi L, Wang D, Chu WC, et al. Volume-based morphometry of brain MR images in adolescent idiopathic scoliosis and healthy control subjects. AJNR Am J Neuroradiol 2009;30:1302-07 CrossRef Medline

8. Wang D, Shi L, Chu WC, et al. Abnormal cerebral cortical thinning pattern in adolescent girls with idiopathic scoliosis. Neuroimage 2012;59:935-42 CrossRef Medline

9. Liu T, Chu WC, Young G, et al. MR analysis of regional brain volume in adolescent idiopathic scoliosis: neurological manifestation of a systemic disease. J Magn Reson Imaging 2008;27: 732-36 CrossRef Medline

10. Wang D, Shi L, Chu WC, et al. A comparison of morphometric techniques for studying the shape of the corpus callosum in adolescent idiopathic scoliosis. Neuroimage 2009;45:738-48 CrossRef Medline

11. Goldberg CJ, Dowling FE, Fogarty EE, et al. Adolescent idiopathic scoliosis and cerebral asymmetry: an examination of a nonspinal perceptual system. Spine (Phila Pa 1976) 1995;20:1685-91 CrossRef Medline

12. Chu WC, Shi L, Wang D, et al. Variations of semicircular canals orientation and left-right asymmetry in adolescent idiopathic scoliosis (AIS) comparing with normal controls: MR morphometry study using advanced image computation techniques. Research into Spinal Deformities 6 2008;140:333 CrossRef

13. Cheng JC, Castelein RM, Chu WC, et al. Adolescent idiopathic scoliosis. Nat Rev Dis Primers 2015;1:15030 CrossRef Medline

14. Beaulieu $C$. The basis of anisotropic water diffusion in the nervous system: a technical review. NMR Biomed 2002;15:435-55 CrossRef Medline 
15. Joly O, Rousie D, Jissendi P, et al. A new approach to corpus callosum anomalies in idiopathic scoliosis using diffusion tensor magnetic resonance imaging. Eur Spine J 2014;23:2643-49 CrossRef Medline

16. Jbabdi S, Sotiropoulos SN, Savio AM, et al. Model-based analysis of multishell diffusion MR data for tractography: how to get over fitting problems. Magn Reson Med 2012;68:1846-55 CrossRef Medline

17. Smith SM, Jenkinson M, Johansen-Berg H, et al. Tract-based spatial statistics: voxelwise analysis of multi-subject diffusion data. Neuroimage 2006;31:1487-505 CrossRef Medline

18. Yushkevich PA, Piven J, Hazlett HC, et al. User-guided $3 D$ active contour segmentation of anatomical structures: significantly improved efficiency and reliability. Neuroimage 2006;31:1116-28 CrossRef Medline

19. Witelson SF. Hand and sex differences in the isthmus and genu of the human corpus callosum: a postmortem morphological study. Brain 1989;112(Pt 3):799-835 CrossRef Medline

20. Sreedharan RM, Menon AC, James JS, et al. Arcuate fasciculus laterality by diffusion tensor imaging correlates with language laterality by functional MRI in preadolescent children. Neuroradiology 2015; 57:291-97 CrossRef Medline

21. Yeh FC, Verstynen TD, Wang Y, et al. Deterministic diffusion fiber tracking improved by quantitative anisotropy. PLoS One 2013;8: e80713 CrossRef Medline

22. Brodmann K. Vergleichende Lokalisationslehre der Grosshirnrinde in ihren Prinzi-pen Dargestellt auf Grund des Zellenbaus. Leipzig: BarthVerlag; 1909

23. Hecke WV. DTI in neurosurgical planning. In: Van Hecke W, Emsell L, Sunaert S. Diffusion Tensor Imaging. New York: Springer-Verlag; 2016:291-306

24. Hofer S, Frahm J. Topography of the human corpus callosum revisited: comprehensive fiber tractography using diffusion tensor magnetic resonance imaging. Neuroimage 2006;32:989-94 CrossRef Medline

25. Caille S, Sauerwein HC, Schiavetto A, et al. Sensory and motor interhemispheric integration after section of different portions of the anterior corpus callosum in nonepileptic patients. Neurosurgery 2005;57:50 -59; discussion 50-59 CrossRef Medline

26. Fabri M, Pierpaoli C, Barbaresi P, et al. Functional topography of the corpus callosum investigated by DTI and fMRI. World J Radiol 2014; 6:895-906 CrossRef Medline

27. Domenech J, Garcia-Marti G, Marti-Bonmati L, et al. Abnormal ac tivation of the motor cortical network in idiopathic scoliosis demonstrated by functional MRI. Eur Spine J 2011;20:1069-78 CrossRef Medline

28. Fabri M, Polonara G, Del Pesce M, et al. Posterior corpus callosum and interhemispheric transfer of somatosensory information: an fMRI and neuropsychological study of a partially callosotomized patient. J Cogn Neurosci 2001;13:1071-79 CrossRef Medline

29. Chau WW, Chu WC, Lam TP, et al. Anatomical origin of abnormal somatosensory evoked potential (SEP) in adolescent idiopathic scoliosis with different curve severity and correlation with cerebellar tonsillar level determined by MRI. Spine (Phila Pa 1976) 2016;41: E598-604 CrossRef Medline

30. Gazzaniga MS, Freedman H. Observations on visual processes after posterior callosal section. Neurology 1973;23:1126-30 CrossRef Medline

31. Clarke S, Maeder P, Meuli R, et al. Interhemispheric transfer of visual motion information after a posterior callosal lesion: a neuropsychological and fMRI study. Exp Brain Res 2000;132:127-33 CrossRef Medline

32. Catanzariti JF, Salomez E, Bruandet JM, et al. Visual deficiency and scoliosis. Spine 2001;26:48-52 CrossRef Medline

33. Pujol J, Vendrell P, Junqué C, et al. When does human brain development end? Evidence of corpus callosum growth up to adulthood. Ann Neurol 1993;34:71-75 CrossRef Medline

34. Chavarria MC, Sanchez FJ, Chou YY, et al. Puberty in the corpus callosum. Neuroscience 2014;265:1-8 CrossRef Medline

35. Burwell RG, Dangerfield PH, Freeman BJ. Etiologic theories of idiopathic scoliosis: somatic nervous system and the NOTOM escalator concept as one component in the pathogenesis of adolescent idiopathic scoliosis. Stud Health Technol Inform 2008;140: 208-17 Medline

36. Mahaudens P, Mousny M. Gait in adolescent idiopathic scoliosis: kinematics, electromyographic and energy cost analysis. Stud Health Technol Inform 2010;158:101-06 Medline

37. Veraart J, Poot DH, Van Hecke W, et al. More accurate estimation of diffusion tensor parameters using diffusion kurtosis imaging. Magn Reson Med 2011;65:138-45 CrossRef Medline 